\title{
Adult Abdominal Migraine Improved by OnabotulinumtoxinA Injections
}

\author{
Neal Hermanowicz \\ Neurology Department, CHRISTUS-St. Vincent Regional Medical Center, Santa Fe, NM, USA
}

\section{Keywords}

Migraine $\cdot$ Abdominal migraine $\cdot$ Onabotulinum toxinA $\cdot$ Abdominal pain

\begin{abstract}
Abdominal migraine is often regarded as a childhood disorder and less commonly described in adults. However, gastrointestinal symptoms are known to occur to adult migraine patients, and recognition of adult abdominal migraine may facilitate treatment of the recurrent abdominal symptoms and avoidance of unproductive and sometimes invasive therapies. Here, I describe a patient with chronic migraine headaches and recurrent abdominal pain both of which showed sustained improvement after treatment with onabotulinumtoxinA injections.
\end{abstract}

\section{Introduction}

Abdominal migraine has often been regarded as occurring in childhood and has been less frequently reported in adults [1]. The International Headache Society (IHS) describes the symptoms of abdominal migraine as "Recurrent episodic attacks of abdominal pain and/or discomfort, nausea, and/or vomiting, occurring infrequently, chronically, or at predictable intervals that may be associated with migraine [2]." The IHS diagnostic criteria include at least 5 attacks with distinct episodes of abdominal pain and/or nausea and/or vomiting, normal gastrointestinal examination and evaluation, and not attributed to another disorder. Recurrent abdominal symptoms in adult patients with migraine may be a source of substantial distress and disability. Recognition and appropriate treatment of adult abdominal migraine may alleviate symptoms and also prevent unnecessary testing and therapies, including invasive procedures. Previous reports on adult abdominal migraine have identified improvement of abdominal symptoms with migraine preventative 
and acute intervention medications [3,4]. Here, I report a single case of an adult with longstanding, recurrent abdominal symptoms and chronic migraine treated by an injection with onabotulinumtoxinA with marked improvement of both headache and abdominal pain.

\section{Case Report/Case Presentation}

Written informed consent was obtained from the patient for publication of this case report. A 73-year-old woman experienced recurrent headaches and abdominal pain since her teenage years. She described the headaches typically as a nonthrobbing pain around one or both of her eyes, associated with both photo- and phono-phobia, nausea but no vomiting, and present on a daily basis. She has no family history of headache. She described the abdominal pain as located midline beneath the umbilicus, a persistent pain that was neither stabbing nor cramping, an aching that was present daily with a variable severity. She had been diagnosed with endometriosis for which she underwent a surgical procedure in her teens without resolution of the pain. She had 1 uncomplicated pregnancy in her early twenties, during which both the headaches and abdominal pain ceased. However, after delivery of her child, both headaches and abdominal pain resumed, and in her early twenties, she underwent a hysterectomy, again without improvement of the abdominal pain. Later investigation of the abdominal pain detected colonic diverticula. Both abdominal pain and headaches continued, sometimes concurrently but not invariably together. While in her forties, the abdominal pain became persistent and headaches were more frequent. Treatment of the headaches with nortriptyline resulted in reduction of frequency and severity of headaches and also of the abdominal pain. In the past, approximately 5 years, the headaches have become more frequent and severe in pain intensity. Medications at her first visit included nortriptyline $60 \mathrm{mg}$ at bedtime, rizatriptan $10 \mathrm{mg}$ as needed, butalbital/ acetaminophen/caffeine 50/325/40 $\mathrm{mg}$ as needed, temazepam $15 \mathrm{mg}$ at bedtime as needed, hydroxyzine $25 \mathrm{mg}$ as needed, and atorvastatin $20 \mathrm{mg}$ daily. The general and neurological examinations were normal. Prior brain imaging by both CT and MRI was unrevealing. A diagnosis of chronic migraine was established, and she was treated with onabotulinumtoxinA following the protocol from the PREEMPT clinical trial for chronic migraine, 31 injections with 5 units of toxin at each site in the scalp, neck, and shoulders [5]. After the injections, she reported the headaches were reduced in both the frequency and severity, and the abdominal pain had ceased. She has now undergone 5 sets of injections with onabotulinumtoxinA, all following the same protocol and separated by 12-16 weeks. The patient has continued to report marked reduction in headache frequency and severity and markedly reduced to absent abdominal pain. In the 2-3 weeks prior to anticipated treatment with onabotulinumtoxinA, she has sometimes experienced return of headaches but not abdominal pain. She has continued to occasionally use rizatriptan 10 $\mathrm{mg}$ for headache. The use of butalbital/acetaminophen/caffeine 50/325/40 $\mathrm{mg}$ and ondansetron $4 \mathrm{mg}$ has ceased.

\section{Discussion/Conclusion}

Gastrointestinal symptoms with migraine headaches are commonplace, and the association of migraine with GI disorders is well established [6]. The patient in this report meets diagnostic criteria for abdominal migraine. She experienced abdominal pain for decades that had been refractory to both medical management and surgical interventions for a

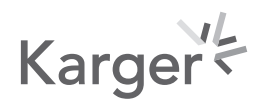


presumed and perhaps erroneous diagnosis of endometriosis. Her abdominal pain largely resolved after initiating onabotulinumtoxinA injections for chronic migraine, and this improvement has been sustained, for the first time in several decades, with her continued toxin injections.

The gut-brain axis has long been of interest, including migraine, although much of the emphasis in prior publications has been on the direction of gut to brain, rather than vice versa [7-9]. Both topiramate and triptan medications have been reported to alleviate abdominal migraine symptoms that are not concurrent with headache $[3,4]$. The injection with onabotulinumtoxinA into the standard locations of the scalp, neck, and shoulders could also disrupt not only the generation of headache but also other symptoms elsewhere, such as the gut, that are centrally mediated by the migraine process.

Calcitonin gene-related peptide (CGRP) has been demonstrated to play a role in pain syndromes, including the headache of migraine [10]. Intravenous infusions of CGRP in human volunteers produced GI symptoms, including abdominal pain [11]. It has been reported that CGRP serum levels are reduced 1 month following onabotulinumtoxinA injection in patients who are favorable responders to this therapy, suggesting a potential CGRP link with both reduction of headache and the abdominal symptoms that may be associated with migraine [12].

This raises several points for consideration. To my knowledge, this is the first case report of a patient with adult abdominal migraine in which the abdominal pain improved with toxin injections. This case also raises questions about the pathogenesis of abdominal migraine as a CGRP-mediated process and the mechanism by which injections of onabotulinumtoxinA into the scalp, neck, and shoulders might alleviate migraine abdominal pain. OnabotulinumtoxinA is described to alleviate chronic migraine headache by peripheral modulation of central nervous system generators of migraine [13]. This CNS modification might address not only the headache component of migraine but also the other extracranialassociated symptoms as well through systemic modulation of CGRP. Previous published observations together with this case report reinforce the idea of migraine as a complex, systemic disorder.

\section{Statement of Ethics}

Written informed consent was obtained from the patient for publication of this case report. This study is exempt from the ethics committee approval. The study is a retrospective description of 1 subject and her experience with migraine and associated symptoms and response to a therapy which is not investigational. The subject was informed well before the manuscript submission that I would like to report her experiences with migraine and abdominal pain, and she was supportive of this.

\section{Conflict of Interest Statement}

The author has received speaking honoraria from Allergan.

\section{Funding Sources}

No funding supported this case report.

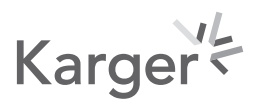




\section{Data Availability Statement}

The data that support the findings of this study are not available. All of the case report information has been obtained from the patient's confidential medical record which cannot be made public. Queries regarding the data in this article should be addressed to Neal Hermanowicz, MD, at neal.hermanowicz@stvin.org, (505) 913-3877, 465 St. Michael's Drive, Suite 116, Santa Fe, NM 87505, USA.

\section{References}

1 Roberts JE, deShazo RD. Abdominal migraine, another cause of abdominal pain in adults. Am J Med. 2012 Nov; 125(11):1135-9.

2 Headache Classification Committee of the International Headache Society. The international classification of headache disorders. Cephalalgia. 2018;38(1):1-211.

3 Woodruff AE, Cieri NE, Abeles J, Seyse SJ. Abdominal migraine in adults: a review of pharmacotherapeutic options. Ann Pharmacother. 2013 Jun;47(6):e27.

4 Moran JA. Adult abdominal migraine and sumatriptan: a case report. Ir Med J. 1998 Dec;91(6):215-6.

5 Aurora SK, Dodick DW, Diener HC, DeGryse RE, Turkel CC, Lipton RB, et al. OnabotulintoxinA for treatment of chronic migraine: results from the double-blind, randomized, placebo-controlled phase of the PREEMPT 1 trial. Cephalalgia. 2010 Jul;30(7):793-803.

6 Arzani M, Jahromi SR, Ghorbani Z, Vahabizad F, Martelletti P, Ghaemi A, et al. Gut-brain axis and migraine headache: a comprehensive review. J Headache Pain. 2020 Feb;21(1):15-2.

7 Mayer EA, Tillisch K. The brain-gut axis in abdominal pain syndromes. Annu Rev Med. 2011;62:381-96.

8 Li BU, Misiewicz L. Cyclic vomiting syndrome: a brain-gut disorder. Gastroenterol Clin North Am. 2002 Sep; 32:997-1019.

9 Scheperjans F, Aho V, Pereira PA, Koskinen K, Paulin L, Pekkonen E, et al. Gut microbiota are related to Parkinson's disease and clinical phenotype. Mov Disord. 2015 Mar;30(3):350-8.

10 Iyengar S, Ossipov MH, Johnson KW. The role of calcitonin gene-related peptide in peripheral and central pain mechanisms including migraine. Pain. 2017 Apr;158(4):543-59.

11 Falkenberg K, Bjerg HR, Olesen J. Two-hour CGRP infusion causes gastrointestinal hyperactivity: possible relevance for CGRP antibody treatment. Headache. 2020 May;60(5):929-37.

12 Cernuda-Morollon E, Ramon C, Martinez-Camblor P, Serrano-Pertierra E, Larrosa D, Pascual J. OnabotulinumtoxinA decreases interictal CGRP plasma levels in patients with chronic migraine. Pain. 2015 May; 156(5): $820-4$.

13 Aurora SK, Brin MF. Chronic migraine: an update on physiology, imaging, and the mechanism of action of two available pharmacologic therapies. Headache. 2016 Jan;57(1):109-25.

\section{Karger'}

